\section{Factores asociados con el índice de masa corporal materno en un grupo de gestantes adolescentes, Medellín, Colombia}

\author{
Factors associated with maternal body mass index \\ in a group of pregnant teenagers, \\ Medellin, Colombia \\ Fatores associados ao índice de massa corporal \\ materno em um grupo de gestantes adolescentes, \\ Medellín, Colômbia
}

Natalia Zapata-López 1

Sandra Lucía Restrepo-Mesa 1
1 Grupo de Investigación Alimentación y Nutrición Humana, Universidad de Antioquia, Antioquia Colombia.

Correspondencia N. Zapata-López Grupo de Investigación Alimentación y Nutrición Humana, Universidad de Antioquia.

Ciudadela Robledo, Carrera 75, no 65-87, Bloque 44, Oficina 109, Medellín, Antioquia

05001000, Colombia.

natyzapata@live.com

\begin{abstract}
The purpose of the study was to assess the influence of socioeconomic risk factors, food security, health, and key anthropometric measures on body mass index (BMI) in a group of teenagers from Medellin, Colombia, in the third trimester of pregnancy. A cross-sectional study was carried out with 294 pregnant teenagers. Data were analyzed using bivariate and multivariate logistic regression analysis. Pregnant teenagers whose families earned less than one minimum wage were more likely to have low weight $(\mathrm{OR}=5.8$; 95\%CI: 1.97-16.8). Age under 15 years was associated with a fourfold increase in low gestational weight. Arm and calf circumference greater than $24 \mathrm{~cm}$ and $32 \mathrm{~cm}$, respectively, were associated with a 94\% reduction in low gestational weight (arm circumference: $O R=0.1 ; 95 \% C I: 0.0-0.2$ ) (calf circumference: $\mathrm{OR}=0.1 ; 95 \% C I$ : 0.0-0.2). In conclusion, low income and young age were associated with low gestational weight. Arm and calf circumference correlated with maternal weight.
\end{abstract}

Nutricional Status; Pregnant Women; Adolescent

\section{Resumen}

El estudio tuvo como objetivo evaluar la influencia de factores socioeconómicos, de seguridad alimentaria, de salud, y algunas medidas antropométricas, en la clasificación del índice de masa corporal (IMC) en un grupo de gestantes adolescentes de la ciudad de Medellín, Colombia, durante el tercer trimestre de gestación. Se realizó un estudio analítico observacional transversal con 294 gestantes. Para el análisis se utilizó un análisis bivariado y un modelo de regresión logística. La mayor probabilidad de presentar bajo peso gestacional la tuvieron aquellas gestantes cuyas familias devengaban menos de un salario mínimo (OR = 5,8; IC95\%: 1,97-16,8). Ser menor de 15 años aumentó cuatro veces la probabilidad bajo peso gestacional y tener un perímetro del brazo y de pantorrilla por encima de $24 \mathrm{~cm}$ y $32 \mathrm{~cm}$, respectivamente, reduce la probabilidad de presentar bajo peso gestacional en un 94\% (perimetro de brazo: OR = 0,1; IC95\%: 0,0$0,2)$; (perímetro de pantorrilla: $O R=0,1$; IC95\%: $0,0-0,2)$. Se concluye que los ingresos y la edad cronológica se asociaron con el bajo peso gestacional. Los perímetros de brazo y pantorrilla se correlacionaron de forma positiva con el peso materno.

Estado Nutricional; Mujeres Embarazadas; Adolescente 


\section{Introducción}

La gestación en la adolescencia es aquella que ocurre en jóvenes entre los 10 y los 19 años y/o cuando se mantiene total dependencia social y económica de los padres 1. En Colombia para el año 2010, la proporción de adolescentes embarazadas alcanzó 19,5\% 2, en el departamento de Antioquia 25,7\%, y en la ciudad de Medellín 23,1\% (Dirección Seccional de Salud de Antioquia. Estadísticas de fecundidad: embarazos 2009. http://www.dssa.gov.co/index.php/estadisticas/fecundidad, accedido el 15/May/2012). En la Red Pública Hospitalaria de Medellín, la cual atiende la población más pobre y vulnerable de la ciudad, las cifras de embarazo adolescente alcanzaron un $34,3 \%$ (fuente: base de datos de la Empresa Social del Estado Metrosalud, consultada el 08/Dic/2010), lo que denota como este es un problema de salud pública, tanto por la alta proporción en el que se presenta, como por sus implicaciones en el estado de salud del binomio madre-hijo y por su significado social, ya que en situaciones socioeconómicas desfavorables perpetúa la pobreza y limita el desarrollo del capita humano del país 3 .

Algunos estudios coinciden en afirmar cómo el estado nutricional de la gestante adolescente y los resultados obstétricos se asocian con factores socioeconómicos, de seguridad alimentaria y nutricional y de salud. Un estudio realizado en México ${ }^{4}$ encontró que el bajo nivel educativo, las edades extremas, la multiparidad, los abortos previos y un inadecuado control prenatal se relacionan con nacimientos prematuros. Por otra parte, un trabajo realizado en el departamento de Antioquia evidencia cómo la precaria situación en la que viven las adolescentes influye sobre el estado nutricional 5 .

El embarazo adolescente se vincula con graves riesgos para la salud, a mayor cercanía de embarazo con el momento de la primera menstruación, mayores pueden ser las complicaciones 6 . En lo nutricional, la adolescente debe satisfacer las necesidades propias y las del hijo 7 lo que representa mayor riesgo de déficit energético y de nutrientes, indispensables para un óptimo incremento de peso de la madre, el buen desarrollo fetal y un adecuado peso al nacer. Las deficiencias nutricionales y el bajo peso al nacer del neonato se han asociado con alteraciones en la formación de las estructuras corporales y con mayor probabilidad de sufrir problemas metabólicos como: obesidad, hipertensión y diabetes en la edad adulta 8 .

En Colombia, según la Encuesta Nacional de la Situación Nutricional (ENSIN) 2010, el bajo peso para las gestantes alcanzó un $16,2 \%$, del cual un $28,6 \%$ se presentó en mujeres menores de 19 años 9 .

De acuerdo con lo anterior, y en vista de la carencia de información que sobre esta problemática se tiene en la ciudad y en el país, el objetivo de este estudio fue evaluar la influencia de factores socioeconómicos, de seguridad alimentaria, de salud, y algunas medidas antropométricas, con la clasificación del índice de masa corporal (IMC) de un grupo de adolescentes en el tercer trimestre de gestación, participantes del programa de control prenatal de la red pública hospitalaria de Medellín.

\section{Metodología}

Se realizó un estudio analítico observacional de corte transversal, que incluyó gestantes adolescentes entre 13 y 19 años de edad, en el tercer trimestre de gestación (semana 27-40), con embarazo monofetal, que realizaron sus controles prenatales en la red pública hospitalaria de Medellín entre agosto de 2011 y marzo de 2012.

La población de referencia fue 1.763 gestantes adolescentes, inscritas en el control prenatal de enero a junio de 2011. Para estimar la proporción de madres en el tercer trimestre de gestación se utilizaron las cifras del programa municipal Buen Comienzo Había una Vez (fuente: base de datos del Programa Buen Comienzo Había Una Vez, consultado el 02/Jun/2011), población que comparte características similares con las gestantes del estudio, la proporción estimada fue $37,3 \%$, cifra cercana al promedio nacional $(31,8 \%) 9$. De acuerdo con lo anterior, la población objetivo fue 659 embarazadas en el tercer trimestre de gestación. La muestra calculada fue 283 madres, la cual se determinó con un error de muestreo de un $4 \%$ y un nivel de confianza de un $95 \%$, se tomó como variable de interés el bajo peso entre gestantes de 13 a 18 años, que según la ENSIN 2010, fue $28,6 \% 9$. Por razones de disponibilidad se evaluaron 294 gestantes, cuya selección no fue aleatoria.

El diseño de la muestra fue estratificado, proporcional y representativo por Unidad Prestadora de Servicios de Salud (UPSS), la cual comprende una unidad hospitalaria y sus centros de salud adscritos.

El estudio indagó por variables demográficas y socioeconómicas: edad, escolaridad, estado civil, lugar y zona de residencia, situación de desplazamiento, ocupación, número de habitantes del hogar, ingresos mensuales, personas encargadas de la manutención del hogar, condiciones de la vivienda y estrato socioeconómico (índice con el cual se representan las caracte- 
rísticas físico exteriores y locativas de una o un grupo de viviendas; el estrato uno se clasifica como bajo-bajo, el dos como bajo, el tres como medio-bajo) ${ }^{10}$. Variables de seguridad alimentaria y nutricional del hogar, de salud (edad de la menarca, edad ginecológica - diferencia en años entre la edad en el momento del embarazo y la primera menstruación: se determinó mayor riesgo en aquellas adolescentes que tuvieron una edad ginecológica menor a cinco años 11), antecedentes obstétricos, planeación y deseo de la gestación, acceso oportuno al control prenatal, enfermedades anteriores y actuales, consumo de alcohol, cigarrillos y/o alucinógenos y; variables antropométricas (estatura, peso actual, IMC actual, perímetro de brazo y perímetro de pantorrilla).

Los datos fueron tomados por un grupo de nutricionistas dietistas, químicos farmacéuticos y estudiantes de prácticas de nutrición y dietética, capacitados y estandarizados para tal fin. Para realizar la entrevista con la gestante, estas fueron contactadas telefónicamente o se captaron en los centros de salud, se les explicaba el proyecto y se invitaban a participar, se realizó la revisión de la calidad de los datos, en caso de inconsistencias se contactó telefónicamente para recuperarlo y/o rectificarlo. Para evaluar el nivel de seguridad alimentaria de los hogares, se empleó la Escala Latinoamericana y Caribeña para la medición de la seguridad alimentaria y nutricional, adaptada y validada para Colombia por Álvarez et al. ${ }^{2}$, utilizada en varios estudios en el ámbito nacional 13 e internacional 14. Las medidas antropométricas fueron tomadas por cuatro nutricionistas dietistas, previamente estandarizadas, utilizando las técnicas de medición de uso internacional 15.

El peso de la gestante se midió en kg con una báscula electrónica marca Tanita HD (Tanita, Tokio, Japón) 313 de 0,1kg de precisión y capacidad de 150kg; la estatura se tomó en centímetros, con un estadiómetro marca Seca (Seca, Hamburgo, Alemania) de $0,1 \mathrm{~cm}$ de precisión y capacidad de $220 \mathrm{~cm}$, y los perímetros, con una cinta métrica marca Mabis (Mabis, Buenos Aires, Argentina) de $0,1 \mathrm{~cm}$ de precisión y capacidad de $150 \mathrm{~cm}$. La clasificación del estado nutricional actual se realizó, según la propuesta de Atalah et al. 16, la cual lo evalúa por medio del IMC -que se obtiene al dividir el peso en $\mathrm{kg}$ sobre la estatura en metros cuadrados -ajustado a las semanas de gestación, clasificándolas como enflaquecidas (IMC < $20 \mathrm{~kg} / \mathrm{m}^{2}$, el cual se entiende también como bajo peso gestacional), con peso adecuado (IMC $20-24,9 \mathrm{~kg} / \mathrm{m}^{2}$ ), sobrepeso (IMC $25-29,9 \mathrm{~kg} / \mathrm{m}^{2}$ ) y obesas (IMC $\geq 30 \mathrm{~kg} / \mathrm{m}^{2}$ ). Se utilizó está propuesta, ya que al ser comparada con otras utilizadas en América Latina, evidenció su mayor exactitud diagnóstica 17 y por no disponer de parámetros nacionales para el monitoreo del incremento de peso en la embarazada adolescente.

Para la clasificación de las medidas de perímetro de brazo y de pantorrilla se utilizaron como puntos de corte: bajo $<24 \mathrm{~cm} 18 \mathrm{y}<32 \mathrm{~cm} 19$, respectivamente.

\section{Análisis de datos}

El procesamiento de datos se realizó en el software estadístico SPSS versión 18.0 (SPSS Inc., Chicago, Estados Unidos). El análisis descriptivo para las variables cuantitativas incluyó medidas de tendencia central como media, mediana, moda y como medida de dispersión la desviación estándar; para las variables cualitativas se utilizaron frecuencias absolutas y porcentajes. Se realizó un análisis bivariado entre la clasificación del IMC y variables explicativas sociodemográficas, de salud y la clasificación de la seguridad alimentaria, para lo cual se utilizaron las pruebas $\chi^{2}$ y correlación de Spearman, según el tipo de variables a analizar y distribución de datos.

Con el test de Kolmogorov-Smirnov se determinó la normalidad de las variables cuantitativas: IMC, perímetro de brazo y de pantorrilla, según su distribución se aplicó la prueba de análisis de varianza y la t de Student para comparar las diferencias de las medidas antropométricas entre grupos. Se realizó una regresión logística binaria global para explorar la asociación entre las variables de estudio con la clasificación de enflaquecidas, según IMC, lo que permitió el cálculo de las razones de disparidad con sus respectivos intervalos de confianza. Las variables que se incluyeron en este modelo fueron las que resultaron ser significativas en el análisis bivariado y aquellas que la literatura informa que están relacionadas con el IMC materno.

\section{Aspectos éticos}

Esta investigación cumplió con los principios éticos para la investigación en humanos, de acuerdo con la declaración de Helsinki y la resolución 008430 del 4 de octubre de 1993 del Ministerio de Salud de Colombia 20. Fue aprobada por los comités de ética para la investigación de la red pública hospitalaria de Medellín, según acta 052011 del 17 de mayo 2011 y de la Sede de Investigación Universitaria (SIU) de la Universidad de Antioquia, según resolución 11-43366 del 14 de julio de 2011. Todas las gestantes dieron su aprobación y quienes acudieron formalizaron su consentimiento para participar. 


\section{Resultados}

$\mathrm{Al}$ analizar las características de las gestantes adolescentes, con relación a la edad, se encontró que la edad promedio fue 17,3 $\pm 1,5$ años, la gestante más joven tuvo 13 años, alrededor de una sexta parte tuvieron 15 años o menos. En el momento de la entrevista un $71,4 \%$ no se encontraban estudiando, de estas un poco más de la mitad manifestó no hacerlo por el embarazo y un $43,8 \%$ por otros motivos $(46,7 \%$ lo denominaron "pereza" o porque no le gustaba estudiar y $6,5 \%$ por dificultades económicas). Por ocupación, una tercera parte no tenían ninguna ocupación actual. En cuanto a la tipología de familia, se resalta que la mayor proporción (50\%) fueron extensas, para las familias monoparentales llama la atención que un $98 \%$ reportaron ser de jefatura femenina (Tabla 1).

El promedio de habitantes del hogar fue cinco personas y $52,7 \%$ de estos eran sustentados económicamente por dos o tres miembros, cuyos empleos se caracterizaron por ser informales y de baja remuneración económica. Se resalta que ocho de cada 10 participantes dependían económicamente de sus familias, la media del ingreso mensual familiar fue 434 dólares estadounidenses $(\$ 766,807$ Col.) y un $39,6 \%$ de los hogares devengaron menos de un salario mínimo mensual legal vigente (USD 321 - \$566,700 Col.). Al medir el nivel de seguridad alimentaria y nutricional en las familias, una de cada seis gestantes se encontraron en inseguridad alimentaria y nutricional, $42,2 \%$ de ellas en leve, $14,5 \%$ en moderada y $8,7 \%$

Tabla 1

Características demográficas, socioeconómicas y de seguridad alimentaria de las gestantes adolescentes en el tercer trimestre de gestación.

\begin{tabular}{|c|c|c|}
\hline \multirow[t]{2}{*}{ Características } & \multicolumn{2}{|c|}{ Total } \\
\hline & $\mathbf{n}$ & $\%$ \\
\hline \multicolumn{3}{|l|}{ Edad (años) } \\
\hline$\leq 15$ & 46 & 15,6 \\
\hline$>15$ & 248 & 84,4 \\
\hline \multicolumn{3}{|l|}{ Nivel educativo } \\
\hline Ninguno/Primaria & 29 & 9,9 \\
\hline Secundaria incompleta/completa & 239 & 81,3 \\
\hline Técnicos o superiores incompletos/completos & 26 & 8,8 \\
\hline \multicolumn{3}{|l|}{ Estado civil } \\
\hline Solteras & 171 & 58,2 \\
\hline Casada/Unión libre & 123 & 41,8 \\
\hline \multicolumn{3}{|l|}{ Zona de residencia } \\
\hline Urbana & 279 & 94,9 \\
\hline Rural & 15 & 5,1 \\
\hline \multicolumn{3}{|l|}{ Desplazamiento en el curso de la gestación } \\
\hline Sí & 30 & 10,2 \\
\hline No & 264 & 89,8 \\
\hline \multicolumn{3}{|l|}{ Motivos del desplazamiento } \\
\hline Violencia & 18 & 60,0 \\
\hline Invierno & 5 & 16,7 \\
\hline Otros motivos & 7 & 23,3 \\
\hline \multicolumn{3}{|l|}{ Ocupación } \\
\hline Estudiante & 75 & 25,5 \\
\hline Ama de casa & 108 & 36,7 \\
\hline Algún tipo de empleo & 16 & 5,4 \\
\hline Ninguna ocupación actual & 95 & 32,3 \\
\hline
\end{tabular}

(continúa) 


\begin{tabular}{|c|c|c|}
\hline \multirow[t]{2}{*}{ Características } & \multicolumn{2}{|c|}{ Total } \\
\hline & $\mathrm{n}$ & $\%$ \\
\hline \multicolumn{3}{|l|}{ Tipología familiar } \\
\hline Nuclear & 71 & 24,1 \\
\hline Monoparenteral & 57 & 19,4 \\
\hline Extensa & 147 & 50,0 \\
\hline Unipersonal/Otra & 19 & 6,5 \\
\hline \multicolumn{3}{|l|}{ Tenencia de la vivienda } \\
\hline Propia/Familiar & 127 & 43,2 \\
\hline Prestada & 27 & 9,2 \\
\hline Arrendada & 125 & 42,5 \\
\hline Invasión/Inquilinato/Otra & 15 & 5,1 \\
\hline \multicolumn{3}{|c|}{ Número de habitantes del hogar } \\
\hline $1-3$ & 73 & 24,8 \\
\hline $4-6$ & 152 & 51,7 \\
\hline$\geq 7$ & 69 & 23,5 \\
\hline \multicolumn{3}{|l|}{ Dependencia económica } \\
\hline Independientes & 56 & 19,0 \\
\hline Dependientes & 238 & 81,0 \\
\hline \multicolumn{3}{|l|}{ Estrato socioeconómico } \\
\hline Uno & 101 & 34,4 \\
\hline Dos & 133 & 45,2 \\
\hline Tres & 60 & 20,4 \\
\hline \multicolumn{3}{|c|}{ Ingresos mensuales de la familia (salarios mínimos mensuales legales vigentes) } \\
\hline$<1$ & 89 & 39,6 \\
\hline $1-2$ & 109 & 48,4 \\
\hline$>2$ & 12 & 5,3 \\
\hline No sabe & 15 & 6,7 \\
\hline \multicolumn{3}{|l|}{ Seguridad alimentaria } \\
\hline Hogares seguros & 100 & 34,6 \\
\hline Hogares inseguros & 189 & 65,4 \\
\hline
\end{tabular}

en severa. Ocho de cada diez hogares fueron estratos socioeconómicos uno y dos (Tabla 1).

Respecto a las características de salud, la edad promedio de la menarca fue 12,4 años, con una edad ginecológica media de 4,4 años, tuvieron una edad ginecológica menor de cinco años $52,1 \%$. El ingreso al control prenatal fue en promedio a la semana $14,8 \pm 6,8$ y se encontró que un $48,6 \%$ lo inició de manera tardía (segundo y tercer trimestre), el número promedio de control prenatal fue cinco (Tabla 2).

En lo referente al planeación un 77,2\% de las gestaciones no fueron planeadas, sin embargo, una de cada siete adolescentes no planificaban. Con relación a la presencia de patologías, un $85,7 \%$ presentaron alguna en cualquier momento del embarazo, la de mayor prevalencia fue la infección vaginal y/o urinaria (89\%), seguida por la ferropenia (44\%) y la anemia $(17,1 \%)$. El consu- mo de alcohol, cigarrillos y sustancias alucinógenas antes del embarazo fue referido por un $66,3 \%$ de las participantes y durante este la prevalencia alcanzó a un 35,4\% (Tabla 2).

Para el indicador de IMC actual, la media fue $25,1 \pm 3,5 \mathrm{~kg} / \mathrm{m}^{2}$, se clasificaron como enflaquecidas un $33,7 \%$, con IMC adecuado $52 \%$ y con sobrepeso u obesidad 14,3\%. Al asociar las variables de estudio con la clasificación del IMC materno, se evidenció que el bajo peso en las gestantes menores de 15 años alcanzó un 52,2\%, con diferencias significativas por grupos de edad ( $\mathrm{p}=0,011$ ) (Tabla 3). El IMC promedio en el grupo de gestantes < 15 años fue un $23,9 \mathrm{~kg} / \mathrm{m}^{2}$, mientras que para el grupo de $\geq 15$ años fue 25,3 $\mathrm{kg} / \mathrm{m}^{2}(\mathrm{p}=0,013)$.

El bajo peso se presentó en mayor proporción en las gestantes cuyas familias devengaron menos de un salario mínimo mensual legal 
Tabla 2

Características de salud y antropométricas de las gestantes adolescentes en el tercer trimestre de gestación

\begin{tabular}{|c|c|c|}
\hline \multirow[t]{2}{*}{ Características } & \multicolumn{2}{|c|}{ Total } \\
\hline & $\mathrm{n}$ & $\%$ \\
\hline \multicolumn{3}{|l|}{ Edad ginecológica (años) } \\
\hline$<5$ & 150 & 52,1 \\
\hline$\geq 5$ & 138 & 47,9 \\
\hline \multicolumn{3}{|l|}{ Antecedentes obstétricos } \\
\hline Ningún antecedente (primigestantes) & 244 & 83,0 \\
\hline Gestaciones previas & 50 & 17,0 \\
\hline Abortos & 24 & 48,0 \\
\hline Nacidos vivos & 26 & 52,0 \\
\hline Periodo intergenésico $<24$ meses & 11 & 42,3 \\
\hline Periodo intergenésico $\geq 24$ meses & 15 & 57,7 \\
\hline Peso al nacer del último hijo $2.500-2.999 \mathrm{~g}$ & 8 & 30,8 \\
\hline Peso al nacer del último hijo 3.000-4.000g & 14 & 53,8 \\
\hline No recuerda el peso al nacer del último hijo & 4 & 15,4 \\
\hline \multicolumn{3}{|l|}{ Gestación actual } \\
\hline \multicolumn{3}{|l|}{ Planeamiento del embarazo } \\
\hline Sí & 67 & 22,8 \\
\hline No & 227 & 77,2 \\
\hline \multicolumn{3}{|l|}{ Deseo del embarazo } \\
\hline Sí & 269 & 91,5 \\
\hline No & 25 & 8,5 \\
\hline \multicolumn{3}{|l|}{ Planificación antes del embarazo } \\
\hline Sí & 72 & 24,5 \\
\hline No & 222 & 75,5 \\
\hline \multicolumn{3}{|l|}{ Acceso al control prenatal } \\
\hline Primer trimestre & 150 & 51,4 \\
\hline Segundo trimestre & 126 & 42,4 \\
\hline Tercer trimestre & 18 & 6,2 \\
\hline \multicolumn{3}{|l|}{ Presencia de enfermedades antes del embarazo } \\
\hline Sí & 102 & 34,7 \\
\hline No & 192 & 65,3 \\
\hline \multicolumn{3}{|l|}{ Presencia de enfermedades durante el embarazo } \\
\hline Sí & 252 & 85,7 \\
\hline No & 40 & 13,6 \\
\hline \multicolumn{3}{|l|}{ Consumo de alcohol, cigarrillos y alucinógenos } \\
\hline Consumo de alcohol, cigarrillos y alucinógenos antes del embarazo & 195 & 66,3 \\
\hline Consumo de alcohol, cigarrillos y alucinógenos durante el embarazo & 104 & 35,4 \\
\hline \multicolumn{3}{|l|}{ Evaluación antropométrica } \\
\hline \multicolumn{3}{|l|}{ Clasificación del estado nutricional actual medido por índice de masa corporal } \\
\hline Bajo peso & 99 & 33,7 \\
\hline Peso adecuado & 153 & 52 \\
\hline Sobrepeso y obesidad & 42 & 14,3 \\
\hline \multicolumn{3}{|l|}{ Clasificación de riesgo según perímetro de brazo } \\
\hline Sin riesgo $(\geq 24)$ & 224 & 76,2 \\
\hline Con riesgo $(<24)$ & 70 & 23,8 \\
\hline \multicolumn{3}{|l|}{ Clasificación de riesgo según perímetro de pantorrilla } \\
\hline Sin riesgo $(\geq 32)$ & 222 & 75,5 \\
\hline Con riesgo $(<32)$ & 72 & 24,5 \\
\hline
\end{tabular}


Tabla 3

Características socioeconómicas, de seguridad alimentaria y de salud, según índice de masa corporal (IMC) de las gestantes adolescentes en el tercer trimestre de gestación.

\begin{tabular}{|c|c|c|c|c|c|c|c|c|}
\hline \multirow[t]{3}{*}{ Características } & \multicolumn{6}{|c|}{ IMC } & \multirow[t]{3}{*}{ Total } & \multirow{3}{*}{$\begin{array}{l}\text { Valor } \\
\text { de } p\end{array}$} \\
\hline & \multicolumn{2}{|c|}{ Bajo peso } & \multicolumn{2}{|c|}{ Peso adecuado } & \multicolumn{2}{|c|}{$\begin{array}{c}\text { Sobrepeso/ } \\
\text { Obesidad }\end{array}$} & & \\
\hline & $\mathbf{n}$ & $\%$ & $\mathrm{n}$ & $\%$ & $\mathrm{n}$ & $\%$ & & \\
\hline \multicolumn{9}{|l|}{ Edad cronológica (años) } \\
\hline$\leq 15$ & 24 & 52,2 & 19 & 41,3 & 3 & 6,5 & 46 & 0,011 * \\
\hline$>15$ & 75 & 30,2 & 134 & 54,0 & 39 & 15,7 & 248 & \\
\hline \multicolumn{9}{|l|}{ Nivel educativo } \\
\hline Ninguno/Primaria & 10 & 34,5 & 15 & 51,7 & 4 & 13,8 & 29 & $0,529 *$ \\
\hline Secundaria & 81 & 33,9 & 126 & 52,7 & 32 & 13,4 & 239 & \\
\hline Técnicos o superiores & 8 & 30,8 & 12 & 46,2 & 6 & 23,1 & 26 & \\
\hline \multicolumn{9}{|l|}{ Estado civil } \\
\hline Soltera & 58 & 33,7 & 88 & 51,2 & 26 & 15,1 & 172 & 0,877 * \\
\hline Casada/Unión libre & 41 & 33,6 & 65 & 53,3 & 16 & 13,1 & 122 & \\
\hline \multicolumn{9}{|l|}{ Ocupación } \\
\hline Estudiante & 25 & 33,3 & 36 & 48,0 & 14 & 18,7 & 75 & 0,667 * \\
\hline Ama de casa & 33 & 30,6 & 61 & 56,5 & 14 & 13,0 & 108 & \\
\hline Algún tipo de empleo & 7 & 43,8 & 6 & 37,5 & 3 & 18,8 & 16 & \\
\hline Ninguna & 34 & 35,8 & 50 & 52,6 & 11 & 11,6 & 95 & \\
\hline \multicolumn{9}{|l|}{ Estrato socioeconómico } \\
\hline Estrato 1 & 33 & 32,7 & 56 & 55,4 & 12 & 11,9 & 101 & 0,654 * \\
\hline Estrato 2 & 47 & 35,3 & 68 & 51,1 & 18 & 13,5 & 133 & \\
\hline Estrato 3 & 19 & 31,7 & 29 & 48,3 & 12 & 20,0 & 60 & \\
\hline \multicolumn{9}{|c|}{ Salario mínimo mensual legal vigente } \\
\hline$<1$ & 39 & 43,8 & 41 & 46,1 & 9 & 10,1 & 89 & 0,003 *x \\
\hline $1-2$ & 34 & 31,2 & 61 & 56,0 & 14 & 12,8 & 109 & \\
\hline$>2$ & 4 & 14,8 & 16 & 59,3 & 7 & 25,9 & 27 & \\
\hline \multicolumn{9}{|l|}{ Desplazamiento } \\
\hline Sí & 13 & 43,3 & 16 & 53,3 & 1 & 3,3 & 30 & 0,154 * \\
\hline No & 86 & 32,6 & 137 & 51,9 & 41 & 15,5 & 264 & \\
\hline \multicolumn{9}{|l|}{ Seguridad alimentaria } \\
\hline Seguridad alimentaria & 31 & 31,0 & 51 & 51,0 & 18 & 18 & 100 & 0,373 * \\
\hline Inseguridad alimentaria & 67 & 35,4 & 99 & 52,9 & 23 & 12 & 189 & \\
\hline \multicolumn{9}{|l|}{ Edad ginecológica (años) } \\
\hline$<5$ & 62 & 41,3 & 74 & 49,3 & 14 & 9,3 & 150 & 0,004 * \\
\hline$\geq 5$ & 36 & 26,1 & 74 & 53,6 & 28 & 20,3 & 138 & \\
\hline \multicolumn{9}{|l|}{ Multiparidad } \\
\hline Sí & 16 & 32,0 & 28 & 56,0 & 6 & 12,0 & 50 & 0,798 * \\
\hline No & 83 & 34,0 & 125 & 51,2 & 36 & 14,8 & 244 & \\
\hline \multicolumn{9}{|c|}{ Periodo intergenésico (meses) } \\
\hline$<24$ & 2 & 18,2 & 8 & 72,7 & 1 & 9,1 & 11 & 0,869 * \\
\hline$\geq 24$ & 4 & 26,7 & 10 & 66,7 & 1 & 6,7 & 15 & \\
\hline \multicolumn{9}{|c|}{ Trimestre de ingreso en el control prenatal } \\
\hline Primero & 57 & 37,7 & 71 & 47,0 & 23 & 15,2 & 151 & 0,061 *x \\
\hline Segundo & 38 & 30,9 & 66 & 53,7 & 19 & 15,4 & 123 & \\
\hline Tercero & 4 & 20,0 & 16 & 80,0 & 0 & 0,0 & 20 & \\
\hline
\end{tabular}

(continúa) 


\begin{tabular}{|c|c|c|c|c|c|c|c|c|}
\hline \multirow[t]{4}{*}{ Características } & \multicolumn{6}{|c|}{ IMC } & \multirow[t]{4}{*}{ Total } & \multirow{4}{*}{$\begin{array}{l}\text { Valor } \\
\text { de } p\end{array}$} \\
\hline & \multirow{2}{*}{\multicolumn{2}{|c|}{ Bajo peso }} & \multirow{2}{*}{\multicolumn{2}{|c|}{ Peso adecuado }} & \multirow{2}{*}{\multicolumn{2}{|c|}{$\begin{array}{l}\text { Sobrepeso/ } \\
\text { Obesidad }\end{array}$}} & & \\
\hline & & & & & & & & \\
\hline & $\mathbf{n}$ & $\%$ & $\mathbf{n}$ & $\%$ & $\mathbf{n}$ & $\%$ & & \\
\hline \multicolumn{9}{|c|}{ Presencia de enfermedad actual } \\
\hline Sí & 85 & 33,7 & 130 & 51,6 & 37 & 14,7 & 252 & 0,876 * \\
\hline No & 14 & 33,3 & 23 & 54,8 & 5 & 11,9 & 42 & \\
\hline \multicolumn{9}{|c|}{ Presencia de infecciones } \\
\hline Sí & 59 & 30,4 & 106 & 54,6 & 29 & 14,9 & 194 & 0,116 * \\
\hline No & 26 & 44,8 & 24 & 41,4 & 8 & 13,8 & 58 & \\
\hline \multirow{2}{*}{\multicolumn{9}{|c|}{$\begin{array}{l}\text { Consumo de sustancias }{ }^{\star * \star} \text { antes del } \\
\text { embarazo }\end{array}$}} \\
\hline & & & & & & & & \\
\hline Sí & 57 & 29,2 & 108 & 55,4 & 30 & 15,4 & 195 & 0,077 * \\
\hline No & 42 & 42,4 & 45 & 45,5 & 12 & 12,1 & 99 & \\
\hline \multirow{2}{*}{\multicolumn{9}{|c|}{ 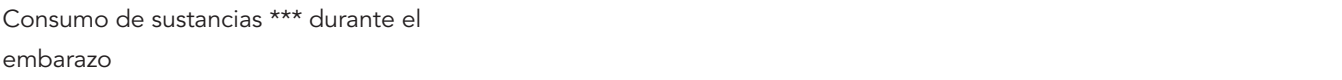 }} \\
\hline & & & & & & & & \\
\hline Sí & 30 & 28,8 & 59 & 56,7 & 15 & 14,4 & 104 & 0,407 * \\
\hline No & 69 & 36,3 & 94 & 49,5 & 27 & 14,2 & 190 & \\
\hline
\end{tabular}

* Prueba de chi cuadrado;

** Correlación de Spearman;

*** Cigarrillos, alcohol y sustancias psicoactivas.

vigente $(p=0,003)$ y las que tuvieron una edad ginecológica menor de cinco años $(p=0,004)$. Esta investigación no encontró asociación entre la clasificación del estado nutricional actual con características demográficas y de salud como estado civil, nivel educativo, ocupación, presencia de infecciones durante el embarazo, periodo intergenésico y seguridad alimentaria $(\mathrm{p}>0,05)$ (Tabla 3). Sin embargo, con relación a este último punto, llama la atención que de las 25 gestantes en inseguridad alimentaria severa, un poco más de la mitad se clasificaron como enflaquecidas.

Para las medidas de perímetro de brazo y de pantorrilla, los valores promedio fueron $26,0 \pm$ $2,9 \mathrm{~cm}$ y $33,9 \pm 2,97 \mathrm{~cm}$, respectivamente. En el caso del perímetro de brazo, un $23,8 \%$ de las participantes se ubicaron por debajo del punto de corte, en el perímetro de pantorrilla la proporción aumentó ligeramente a $24,5 \%$; para ambas medidas hubo una diferencia significativa entre el promedio y la clasificación del IMC materno $(p=0,000)$. Para el caso del perímetro de brazo, las gestantes con IMC bajo tuvieron $3,1 \mathrm{~cm}$ menos que las gestantes con IMC adecuado, este comportamiento fue similar para el perímetro de pantorrilla, ya que las gestantes con IMC bajo presentaron $3 \mathrm{~cm}$ menos que las gestantes con IMC adecuado (Tabla 4).

$\mathrm{Al}$ aplicar el modelo de regresión logística, se encontró que tener un perímetro de brazo y de pantorrilla por encima de $24 \mathrm{~cm}$ y de $32 \mathrm{~cm}$, respectivamente, reduce la probabilidad de presentar bajo peso gestacional en un $94 \%$ (perímetro de brazo: OR = 0,1; IC95\%: 0,0-0,2); (perímetro de pantorrilla: $\mathrm{OR}=0,1$; IC95\%: 0,0-0,2). La mayor probabilidad de presentar bajo peso la tuvieron aquellas gestantes cuyas familias devengaban menos de un salario mínimo mensual legal vigente (OR $=5,8$; IC95\%: 1,97-16,8), además tener 15 años o ser menor, aumentó cuatro veces la probabilidad de presentar bajo peso al nacer (Tabla 5).

\section{Discusión}

Los hallazgos de este estudio indican que los ingresos económicos del hogar, la edad cronológica y la edad ginecológica se asociaron con el bajo peso gestacional. Un ingreso familiar inferior a un salario mínimo mensual legal vigente, y tener 15 años o menos, aumentan el riesgo de presentar bajo peso durante el embarazo. Las medidas de perímetro de brazo y de pantorrilla se correlacionaron con el peso materno. Las gestantes con una edad ginecológica menor a cinco años fueron las que presentaron la mayor proporción de bajo peso gestacional.

El embarazo en adolescentes es un grave problema de salud pública, las mayores tasas se 
Tabla 4

Promedio de los perímetros de brazo y pantorrilla, según índice de masa corporal (IMC) de las gestantes adolescentes en el tercer trimestre de gestación.

\begin{tabular}{lcccc}
\hline Medida & \multicolumn{2}{c}{ Clasificación del estado nutricional por IMC } & Valor de $p$ * \\
& Bajo peso & Peso adecuado & $\begin{array}{c}\text { Sobrepeso/ } \\
\text { Obesidad }\end{array}$ & \\
\hline Perímetro de brazo $(X \pm D E)$ & $23,3 \pm 1,4$ & $26,5 \pm 1,7$ & $30,4 \pm 3,0$ & $<0,01$ \\
Perímetro de pantorrilla $(X \pm D E)$ & $31,3 \pm 1,6$ & $34,3 \pm 1,8$ & $38,3 \pm 2,7$ & $<0,01$ \\
\hline
\end{tabular}

DE: desviación estándar; $X$ : promedio.

* Análisis de varianza.

Tabla 5

Asociación del bajo peso gestacional con variables sociodemográficas, económicas, de salud y de seguridad alimentaria.

\begin{tabular}{lcccc}
\hline Variable & $\boldsymbol{\beta}$ & Valor de $\mathbf{p}$ & OR & IC95\% \\
\hline Edad ginecológica & $-0,28$ & 0,604 & 0,75 & $0,26-2,19$ \\
Edad cronológica & 1,39 & 0,049 & 4,03 & $1,00-16,16$ \\
Ingresos por salarios mínimos mensuales legales vigentes & 1,75 & 0,001 & 5,76 & $1,97-16,84$ \\
Clasificación perímetro de brazo & $-2,81$ & 0,000 & 0,06 & $0,02-0,19$ \\
Clasificación perímetro de pantorrilla & $-2,76$ & 0,000 & 0,063 & $0,02-0,22$ \\
Presencia de infecciones durante el embarazo & 0,50 & 0,365 & 1,65 & $0,56-4,85$ \\
Consumo de sustancias * antes del embarazo & 0,35 & 0,493 & 1,42 & $0,52-3,90$ \\
Clasificación seguridad alimentaria & $-0,58$ & 0,294 & 0,56 & $0,19-1,65$ \\
\hline
\end{tabular}

IC95\%: intervalo de confianza de 95\%; OR: odds ratio.

* Cigarrillo, alcohol y sustancias psicoactivas.

presentan en los países en vía de desarrollo, la Organización Mundial de la Salud (OMS) refiere que alrededor de un $90 \%$ de los casos de embarazos adolescentes ocurren en este tipo de países 3 . Como problema de múltiples consecuencias, uno de los elementos a considerar es la deserción escolar, ya que siete de cada diez adolescentes no se encontraban estudiando en el momento de la entrevista, y de estas, un poco más de la mitad no lo hacía debido al embarazo y el porcentaje restante por otros motivos. Organismos internacionales, como la OMS y el Fondo de las Naciones Unidas para la Infancia (UNICEF), concluyen que la maternidad en la adolescencia obstaculiza la continuidad escolar, reduce las oportunidades de inserción laboral y el acceso a un salario digno 21 , lo que desencadena la reproducción intergeneracional de la pobreza 3 .

Este estudio encontró que ocho de cada diez hogares, donde residían las adolescentes, pertenecían a los estratos uno y dos, los cuales se caracterizan por ser los más pobres de la ciudad.
La Comisión Económica para América Latina y el Caribe encontró que la tasa específica de fecundidad adolescente del quintil más pobre es cuatro veces mayor que la del quintil más rico ${ }^{3}$. En este trabajo, el ingreso económico en casi nueve de cada diez familias fue inferior a dos salarios mínimos mensuales legales vigentes, similar a lo encontrado en el perfil alimentario y nutricional de Medellín 22, el cual determinó que este dinero no alcanza para cubrir las necesidades básicas, ni obtener una canasta alimentaria recomendada, ya que para alcanzarla, una familia de cuatro miembros requiere sólo para la compra de alimentos 1,3 salarios mínimos.

Sumado a lo anterior, la inseguridad alimentaria fue superior a la encontrada en la ciudad 22 y en el país 9 , la cual se asocia con pobre calidad de la dieta. En mujeres embarazadas, esta se relaciona con incremento en el riesgo de algunos defectos del nacimiento y mayor probabilidad de niños con bajo peso al nacer 23 . En Colombia, una investigación realizada con este grupo poblacio- 
nal encontró un $22,7 \%$ de los hogares en inseguridad alimentaria severa, de estos, un $38 \%$ de las adolescentes presentaron bajo peso gestacional 24. Al respecto, Pérez y Bernal 25 concluyeron que medir el nivel de seguridad alimentaria puede predecir el estado nutricional de la embarazada. Con relación a estos hallazgos, un estudio realizado en la ciudad de Cali-Colombia encontró cómo la proporción de bajo peso gestacional fue mayor en las mujeres que pertenecían al régimen vinculado y subsidiado en salud 26 , que para el caso del país son la población más pobre, cuyos ingresos mensuales no superan los dos salarios mínimos mensuales legales vigentes.

El gran número de familias extensas y monoparentales de jefatura femenina encontradas en este estudio fue superior a la reportada por Restrepo et al. ${ }^{5}$ y por Álvarez et al. 22 en Colombia. Los hogares con jefatura femenina son más pobres que los de jefatura masculina 5 . Lo anterior demuestra la alta vulnerabilidad social y económica de las gestantes participantes del estudio, condiciones que no favorecen el buen desarrollo de la gestación, ni el bienestar del binomio madre-hijo.

Contrario a los resultados de una investigación realizada en Venezuela 27 , donde cerca de un $80 \%$ de las adolescentes cursaban su embarazo sin la compañía de una pareja, en este trabajo, cuatro de cada diez madres eran casadas o convivían con sus compañeros, cifra similar a la reportada en un estudio realizado en Argentina $28 \mathrm{e}$ inferior a la encontrada en un estudio realizado en el país 29 . Este hallazgo es importante, ya que la literatura sostiene, cómo la presencia de un compañero estable influye de manera positiva en los resultados de la gestación 30 , por ello, la alta proporción de gestantes con compañero que se encontró en este estudio se convierte en un factor protector de su gestación.

En cuanto a las características de salud, llama la atención por lo contradictorio, el hecho de que la mayoría de las gestaciones no fueron planeadas, sin embargo, siete de cada diez adolescentes no planificaban. De la Cuesta 31 expone como la anticoncepción en adolescentes no es un tema que pueda limitarse únicamente a la falta de conocimientos, existe evidencia que sugiere que los adolescentes no siempre están motivados a planificar, bien sea por la invulnerabilidad que perciben o por dificultades para negociar su uso con la pareja. Otros autores plantean cómo la falta de privacidad y miedo a revelar su vida sexual son razones para que las adolescentes no asistan a los servicios de planificación 21 .

Al contrastar los hallazgos respecto a la edad ginecológica, se encontró que un poco más de la mitad de las participantes se clasificaron en ries- go según está variable, ya que cuando esta es menor a cinco años impone un mayor compromiso para la salud y el estado nutricional de la adolescente. Un estudio realizado en Cuba, analizó los resultados maternos y perinatales de la gestación adolescente y encontró mayor probabilidad de presentar enfermedades y riesgos obstétricos en el grupo de adolescentes con edad ginecológica menor a cinco años, ya que tuvieron una proporción más alta de hipertensión inducida por el embarazo, bajo peso gestacional y nacimiento de hijos con peso inferior a $2.500 \mathrm{~g} 11$.

Entre los antecedentes obstétricos, se encontró que casi dos de cada diez gestantes habían tenido un embarazo previo, cifra comparable con otros estudios realizados en el país 29,32 , esta característica de multiparidad representa un mayor riesgo para la gestante adolescente. Un estudio realizado en Brasil, caracterizó demográficamente un grupo de gestantes adolescentes multíparas y encontró que estas tenían un menor nivel educativo, percibían menos ingresos económicos y eran más pobres comparadas con un grupo de adolescentes primigestantes 33 .

Con relación al periodo intergenésico, se encontró que para un $42,3 \%$ fue menor a 24 meses, factor de riesgo adicional, ya que el cuerpo tarda dos años en reponer las reservas de nutrientes y adecuarse para afrontar otra gestación, intervalos intergenésicos cortos están asociados con el incremento de resultados perinatales adversos 34 .

Las patologías de mayor prevalencia fueron la infección vaginal y/o urinaria, la ferropenia y la anemia. Esta última reduce en la madre la capacidad de trabajo e intelectual, incrementa la susceptibilidad a la infección y podría llevar incluso a la muerte. En los países de bajos recursos, el riesgo de presentar esta enfermedad es mayor, probablemente por la combinación de la anemia con enfermedades obstétricas como la hemorragia, infección, entre otras. La anemia severa se asocia con resultados perinatales adversos como parto pretérmino y bebes pequeños para la edad gestacional, además, los hijos de madres anémicas tienen mayor riesgo de desarrollar esta enfermedad durante la infancia, pobre crecimiento físico y mental y función cognitiva disminuida ${ }^{35}$. Durante la gestación en la adolescencia, todos estos riesgos se maximizan 36 , puesto que las necesidades de hierro se encuentran aumentadas como consecuencia de los cambios en el tamaño corporal, la formación de tejidos 37 y las necesidades de hierro adicional, indispensable para la síntesis acelerada de glóbulos rojos, la formación de la placenta y el crecimiento y desarrollo del feto 38 .

La hipertensión y la diabetes se encontraron en menor proporción en el presente estudio, re- 
sultado comparable con otro realizado en Malasia 39 . Pese a no haber encontrado asociación estadística entre el estado de salud y la presencia de infecciones con el peso materno, es de gran importancia clínica y debe llamar la atención del equipo de salud el alto porcentaje de gestantes con infecciones, ya que se ha demostrado su relación con ruptura prematura de membranas, parto pretérmino, bajo peso al nacer, endometritis post-parto, muerte fetal y neonatal 40 .

La alta proporción en el consumo de alcohol, cigarrillos y sustancias alucinógenas fue otro hallazgo relevante, ya que antes del embarazo seis de cada diez gestantes las consumieron y durante este la proporción fue tres de cada diez, cifras alarmantes por los efectos deletéreos en el desarrollo neurológico, psicomotor y crecimiento de los recién nacidos ${ }^{41}$. Omar et al. 40 concluyeron cómo el uso de marihuana, cocaína o estimulantes durante la gestación fue mayor en gestantes más jóvenes, con menor nivel educativo, con más bajos ingresos económicos y con mayor proporción de bajo peso gestacional. Problemática que demanda de acciones efectivas de intervención por las graves consecuencias en la salud infantil. El etanol en la placenta inhibe la síntesis de proteínas y disminuye la trasferencia de glucosa y de aminoácidos hacia el feto, además puede ocasionar el síndrome de alcoholismo fetal, caracterizado por deficiencias en el crecimiento pre y postnatal, retraso mental leve o moderado, microcefalia, trastornos de hiperactividad y déficit de atención ${ }^{42}$. El cigarrillo y las sustancias alucinógenas limitan la corriente de oxígeno desde el útero hasta la placenta, lo que aumenta el estrés materno fetal y, en consecuencia, se aumentan los niveles de catecolaminas, lo que conlleva al retardo del crecimiento intrauterino y bajo peso al nacer 43 .

En cuanto a la clasificación del IMC en tercer trimestre de gestación, la mitad de las participantes presentaron peso adecuado, tres de cada diez bajo peso y $14,3 \%$, sobrepeso y obesidad; la proporción de bajo peso gestacional estuvo cinco puntos porcentuales por encima del encontrado a nivel nacional, según la ENSIN 2010 9, y cercano al encontrado por Sarmiento et al. ${ }^{29}$, donde $31,2 \%$ de las gestantes adolescentes presentaron bajo peso; ambas cifras difieren a lo reportado en otro estudio realizado en el país 32 , donde la proporción de bajo peso gestacional fue superior. En la presente investigación, las gestantes menores de 15 años tuvieron mayor riesgo de presentar bajo peso gestacional que sus contemporáneas mayores. En un estudio realizado en el país Mancilla et al. ${ }^{44}$ coincidieron con estos hallazgos, al reportar diferencias significativas en el valor promedio de IMC por grupo de edad. En la gestación, la desnutrición y el déficit de micronutrientes acarrea graves consecuencias, tanto para el madre, como para el recién nacido, que se empeoran si la gestación ocurre en la adolescencia; en el caso del neonato, este presenta mayor riesgo de bajo peso al nacer, incremento de la tasa de mortalidad infantil, retardo o detención del crecimiento, riesgo de déficit psicomotor 29 y en la vida adulta riesgo de padecer enfermedades crónicas no trasmisibles 8; por otra parte, para la mujer gestante adolescente, el bajo peso conlleva a la restricción de su propio crecimiento, una probabilidad aumentada de padecer anemia y menor capacidad de trabajo 45 .

Al igual que en otros estudios 44,46 éste no encontró relación entre el peso materno con variables de tipo demográfico como estado civil, escolaridad, ocupación, tipología de familia - debido posiblemente a las características socioeconómicas similares en las que viven las gestantes -, tampoco se encontró relación con variables de salud como paridad, periodo intergenésico y presencia de infecciones durante la gestación.

Con relación al perímetro de brazo y de pantorrilla, se evidenció que estas medidas tuvieron un alto nivel predictivo de bajo peso gestacional. La alta correlación con el peso materno demuestra su gran utilidad en la práctica clínica, además los valores promedio no presentan diferencias por trimestre de gestación, lo que las hace muy útiles para tamizar mujeres en edad fértil o gestantes a riesgo nutricional 18,19. La Organización Panamericana de la Salud (OPS), sugiere medir en gestantes el perímetro de brazo para determinar nacimientos de niños con bajo peso al nacer o mortalidad infantil tardía 47 y algunos autores han evidenciado la relación entre perímetro de pantorrilla y baja talla al nacer 48 .

La antropometría es una herramienta sencilla y de bajo costo para la detección de embarazadas a riesgo o con vulnerabilidad social y económica. Se recomienda evaluar el peso y la estatura, el incremento de peso durante la gestación y los perímetros de brazo y pantorrilla como medidas que contribuyen a identificar mujeres con riesgo nutricional.

Los hallazgos de este estudio ratifican que las gestantes adolescentes se enfrentan a complicaciones económicas, sociales y biológicas que se asocian con su estado nutricional, lo que conlleva a graves repercusiones para la madre y el hijo.

Los programas de control prenatal no pueden limitarse a declarar las embarazadas adolescentes cómo "alto riesgo obstétrico", sino que además deben responder a las características biopsicosociales de las adolescentes, ajustándose a su contexto específico y a las particularidades de la identidad femenina, que las motive a 
generar cambios de conducta frente a la sexualidad y a controlar su propia fecundidad 49 . Estos programas deben desarrollar acciones encaminadas a detectar conocimientos y prácticas de riesgo para la salud materno fetal y a monitorear el comportamiento de los mismos en el trascurso de la gestación, que contribuyan a la toma de decisiones oportunas en pro del bienestar de la madre y sus hijos.

Se reconoce como limitante del estudio, que la selección final de las unidades de análisis no fue aleatoria, sin embargo, al haber alcanzado la muestra total y su representatividad por UPSS, permite que los datos puedan ser generalizados a la población objetivo. No fue posible evaluar el indicador de IMC pregestacional, ya que para las adolescentes de 18 años o menos se dispo- ne de cuatro categorías de clasificación y para las de 19 años tres categorías, las cuales no son comparables entre sí. Debido al diseño del estudio, no es posible hablar de la causalidad. $\mathrm{Si}$ bien la muestra no fue representativa por grupo de edad, la proporción de gestantes $\leq$ de 15 años fue pequeña, lo que puede limitar las inferencias realizadas.

Se sugiere el desarrollo de futuras investigaciones que indaguen la relación entre las características socioeconómicas maternas y el producto de la gestación, y de investigaciones cualitativas que contribuyan a comprender las implicaciones de la inseguridad alimentaria y el hambre en la gestación; además de la construcción y diseño de un modelo gráfico para el monitoreo nutricional de las embarazadas adolescentes en el país.

\section{Resumo}

O estudo teve como objetivo avaliar a influência de fatores socioeconômicos, de segurança alimentar, de saúde, e algumas medidas antropométricas, na classificação do índice de massa corporal (IMC) em um grupo de gestantes adolescentes da Cidade de Medel lín, Colômbia, durante o terceiro trimestre de gestação. Realizou-se estudo analítico observacional transversal com 294 gestantes. Utilizou-se análise bivariada e um modelo de regressão logística. As gestantes cujas famílias ganhavam menos de 1 salário mínimo (OR = 5,8; IC95\%: 1,9-16,8) tiveram maior probabilidade de apresentar baixo peso gestacional. Ser menor de 15 anos aumentou quatro vezes a probabilidade de baixo peso gestacional, e ter um perímetro do braço e da panturrilha maior que $24 \mathrm{~cm}$ e $32 \mathrm{~cm}$, respectivamente, reduz em 94\% a probabilidade de apresentar baixo peso gestacional (perímetro do braço: $O R=0,1 ; I C 95 \%$ : 0,0-0,2; perímetro da panturrilha: $O R=0,1 ;$ IC95\%: 0,0-0,2). Conclui-se que a renda e a idade cronológica associaram-se com o baixo peso gestacional. Os perimetros do braço e da panturrilha relacionaram-se de forma positiva com o peso materno.

Estado Nutricional; Gestantes; Adolescente

\section{Colaboradores}

N. Zapata-López y S. L. Restrepo-Mesa participaron en la formulación y ejecución del proyecto, en el análisis de resultados y en la escritura del artículo.

\section{Agradecimientos}

El grupo de investigación agradece a las gestantes adolescentes, sujetos centrales de este proceso por su vinculación al proyecto, a la red pública hospitalaria de Medellín ESE Metrosalud por permitir realizar la investigación en la institución y a todas las instancias financiadores que permitieron hacer viable este proyecto.

Este proyecto fue financiado por El Comité para e Desarollo de la Investigación (CODI) de la Universidad de Antioquia, el Grupo de Investigación en Alimentación y Nutrición Humana con recursos de sostenibilidad de 2009-2010 de la Vicerrectoria de Investigación, ESE Metrosalud y laboratorios Laproff. 


\section{Referencias}

1. Organización Mundial de la Salud. 59a Asamblea Mundial de la Salud: contribución la OMS a la aplicación de la estrategia para la salud y el desarrollo del niño y el adolescente. http://apps.who.int/gb/ ebwha/pdf_files/WHA59-REC3/A59REC3_sp.pdf (accedido el 05/May/2012).

2. Profamilia; Ministerio de la Protección Familiar; Instituto Colombiano de Bienestar Familiar; United States Agency for International Development. Quinta Encuesta Nacional de Demografía y Salud: ENDS-2010. Bogotá: Profamilia; 2011.

3. Gómez P, Molina R, Zamberlin N. Fecundidad en adolescente. In: Orozco LT, editor. Factores relacionados con el embarazo y la maternidad en menores de 15 años en América Latina y el Caribe. Lima: Federación Latinoamericana de Sociedades de Obstetricia y Ginecología; 2010.

4. Osorno L, Rupay GE, Rodríguez J, Lavadores AI, Dávila J, Echeverría M. Factores maternos relacionados con prematuridad. Ginecol Obstet Méx 2008; 76:526-36

5. Restrepo S, Mancilla L, Parra B, Manjares LM, Zapata N, Restrepo P, et al. Evaluación del estado nutricional de mujeres gestantes que participaron en un programa de alimentación y nutrición. Rev Chil Nutr 2010; 37:18-30.

6. Coall D, Chisholm J. Evolutionary perspectives on pregnancy: maternal age at menarche and infant birth weight. Soc Sci Med 2003; 57:1771-81.

7. Jones R, Cederberg H, Wheeler S, Poston I, Hutchinson C, Seed P, et al. Relationship between maternal growth, infant birth weight and nutrient partitioning in teenage pregnancies. Br J Obstet Gynecol 2010; 117:200-11.

8. McMillen C, MacLaughlin S, Muhlhausler B, Gentili S, Duffield J, Morrison J. Developmental origins of adult health and disease: the role of periconceptional and foetal nutrition. Basic Clin Pharmacol Toxicol 2008; 102:82-9.

9. Profamilia; Instituto Nacional de Salud; Instituto Colombiano de Bienestar Familiar, Profamilia; Ministerio de Protección Familiar. Encuesta nacional de la situación nutricional en Colombia 2010: ENSIN. Bogotá: Instituto Colombiano de Bienestar Familiar; 2011.

10. Departamento Administrativo Nacional de Estadística. Estratificación socioeconómica. http:// www.dane.gov.co/index.php?option=com_conte nt\&view=article\&id=354\&Itemid=114 (accedido el May/2012).

11. Balestena Sánchez JM, Balestena Sánchez SG. Impacto de la menarquía en los resultados maternos perinatales en la adolescencia. Rev Cuba Obstet Ginecol 2005; 31:0-0.

12. Álvarez MC, Estrada A, Montoya EC, Melgar-Quiñónez H. Validación de la escala de la seguridad alimentaria domestica en Antioquia - Colombia 2006. Salud Pública Méx 2006; 48:474-81.

13. Cadavid M, Zapata M, Aguirre D, Álvarez MC. Coeficiente intelectual de niños escolarizados en instituciones públicas de las zonas nororiental y noroccidental de Medellín, según el nivel de seguridad alimentaria del hogar y condiciones socioeconómicas. Rev Chil Nutr 2011; 38:392-403.
14. Paras P, Pérez-Escamilla R. Inseguridad alimentaria en México. http://www.vanderbilt.edu/lapop/ mexico/2008-inseguridadalimentaria.pdf (accedido el 15/Abr/2012).

15. Harrison G, Buskirk E, Carter J, Johnston F, Lohman T, Pollock M, et al. Skinfold thicknesses and measurement technique. In: Lohman T, Roche A, Martorell R, editors. Antropometric standardization reference manual. Champaign: Human Kinetics Publishers; 1988. p. 55-70.

16. Atalah E, Castillo C, Castro R, Aldea A. Propuesta de un nuevo estándar de evaluación nutricional de embarazadas. Rev Méd Chile 1997; 125:1429-36.

17. Benjumea MV. Exactitud diagnóstica de cinco referencias gestacionales para predecir el peso insuficiente al nacer. Biomédica 2007; 27:42-55.

18. Benjumea Rincón MV, Bacallao Gallestey J, Jiménez R. La predicción del bajo peso y del peso insuficiente al nacer mediante la antropometría materna. Hacia Promoc Salud 2009; 14:35-53.

19. Benjumea Rincón MV. Antropometría materna como predictora de peso al nacer [Tesis de Doctorado]. La Habana: Instituto Superior de Ciencias Básicas y Preclínicas “Victoria de Girón”; 2007.

20. Ministerio de Salud. Resolución no 008430 de 1993: por la cual se establecen las normas científicas, técnicas y administrativas para la investigación en salud. Bogotá: Ministerio de Salud; 1993.

21. Santos KA. Teenage pregnancy contextualized: understanding reproductive intentions in a Brazilian shantytown. Cad Saúde Pública 2012; 28:655-64

22. Álvarez LE, Mancilla L, González L, Isaza U. Perfil alimentario y nutricional de Medellín 2010. Medellín: Alcaldía de Medellín; 2010.

23. Hromi A, Bermúdez A, Chapman D, Segura S, Damio G, Melgar H, et al. Household food insecurity is associated with depressive symptoms among low-income pregnant Latinas. Matern Child Nutr 2011; 7:421-30.

24. Muñoz MN, Martínez J, Quintero R. Validación de la Escala Latinoamericana y Caribeña de Seguridad Alimentaria en gestantes adolescentes. Rev Salud Pública 2010; 12:173-83.

25. Pérez A, Bernal J. Predicción del estado nutricional mediante variables antropométricas y de seguridad alimentaria en el hogar de un grupo de embarazadas de Caracas, Venezuela. Nutr Hosp 2006; 21:611-6.

26. Murillo OL, Zea MP, Pradilla A. Situación nutricional de la gestante y su recién nacido en Cali, 2008. Rev Salud Pública 2011; 13:585-96.

27. Peña E, Sánchez A, Solano L. Perfil de riesgo nutricional en la adolescente embarazada. Arch Latinoam Nutr 2003; 53:141-9.

28. Gogna M, Binstock G, Fernandez S, Ibarlucia I, Zamberlinc N. Adolescent pregnancy in Argentina: evidence-based recommendations for public policies. Reprod Health Matters 2008; 16:192-201.

29. Sarmiento OL, Ramírez A, Kutschbach BS, Pinzón PL, García S, Olarte AC, et al. Nutrition in Colombian pregnant women. Public Health Nutr 2012; $15: 955-63$ 
30. Balayla J, Azoulay L, Abenhaim HA. Maternal marital status and the risk of stillbirth and infant death: a population-based cohort study on 40 million births in the United States. Womens Health Issues 2011; 21:361-5.

31. De la Cuesta C. Tomarse el amor en serio: contexto del embarazo en la adolescencia. Medellín: Editorial Universidad de Antioquia; 2002.

32. Quintero Tabares R, Muñoz Astudillo N, Álvarez Sierra LE, Medina Perea GA. Estado nutricional y seguridad alimentaria en gestantes adolescentes: Pereira, Colombia, 2009. Invest Educ Enferm 2010; 28:204-13.

33. de Fátima Rato Padin $M$, de Souza e Silva $R$, Chalem E, Mitsuhiro SS, Barros MM, Guinsburg $\mathrm{R}$, et al. Brief report: a socio-demographic profile of multiparous teenage mothers. J Adolesc 2009; 32:715-21.

34. Hussaini KS, Ritenour D, Coonrod DV. Interpregnancy intervals and the risk for infant mortality: a case control study of Arizona infants 2003-2007. Matern Child Health J 2012; [Epub ahead of print].

35. Goonewardene M, Shehata M, Hamad A. Anaemia in pregnancy. Best Pract Res Clin Obstet Gynaecol 2012; 26:3-24.

36. Organización Panamericana de la Salud. La anemia entre adolescentes y mujeres adultas en Amé rica Latina y el Caribe. http://new.paho.org/hq/ dmdocuments/2009/AdolescentAnemiaSpan\%20 (2).pdf (accedido el 05/May/2012).

37. World Bank. Nutrición de los adolescentes: un vistazo. http://siteresources.worldbank.org/INTPHA AG/Resources/AAGAdolHealthSpanApr03.pdf (accedido el 07/May/2012).

38. Parra B. Evaluación bioquímica del estado nutricional del hierro y folato en un grupo de mujeres gestantes participantes del programa MANA para la vida. In: Restrepo S, editor. Alimentación y nutrición de la mujer gestante: diagnóstico y lineamientos para la acción. Medellín: Divegráficas; 2007. p. 217-43.

39. Omar K, Hasim S, Muhammad NA, Jaffar A Hashim SM, Siraj HH. Adolescent pregnancy outcomes and risk factors in Malaysia. Int J Gynaecol Obstet 2010; 111:220-3.

40. Giraldo PC, Araújo ED, Eleutério Jr. J, Amaral RLG, Passos M, Goncalves AK. The prevalence of urogenital infections in pregnant women experiencing preterm and full-term labor. Infect Dis Obstet Gynecol 2012; [Epub ahead of print].
41. Rahu K, Rahu M, Pullmann H, Allik J. Effect of birth weight, maternal education and prenatal smoking on offspring intelligence at school age. Early Hum Dev 2010; 86:493-7.

42. Cuervo L. Alcohol en la gestación y sus consecuencias en las relaciones feto-placentarias. http:// www.javeriana.edu.co/Facultades/Ciencias/neu robioquimica/libros/perinatal/alcoholed.html (accedido el 29/May/2012).

43. Poleta F, López JS, Gili JA, Montalvo G, Castilla E. Consumo y exposición al humo de tabaco en mujeres embarazadas de Ecuador. Rev Panam Salud Pública 2010; 27:56-65.

44. Mancilla LP, Restrepo SL, Estrada A, Manjarés LM, Parra B. Estado nutricional de un grupo de gestantes y su relación con indicadores socioeconómicos y de ingesta dietética. Antioquia, Colombia. Rev Fac Nac Salud Pública 2011; 29:232-40.

45. Luder E, Alton I. Nutrition needs of underweight adolescents. In: Stang J, Story M, editors. Guidelines for adolescent nutrition services. Minneapolis: University of Minnesota; 2005. p. 93-100.

46. Walker LO. Low-income women's reproductive weight patterns empirically based clusters of prepregnant, gestational, and postpartum weights. Womens Health Issues 2009; 19:398-405.

47. Cedillo N, Elias J, Dellán JE, Toro J. Estado nutricional de las adolescentes embarazadas: relación con el crecimiento fetal. Rev Obstet Ginecol 2006; 66:233-40.

48. González-Cossío T, Sanín LE, Hernández M, Rivera J, Hu H. Longitud y peso al nacer: papel de la nutrición materna. Salud Pública Méx 1998; 40:119-26.

49. Carvallo MA, Moreno A. La construcción de la identidad de género y la representación de la maternidad. In: Actas de 4o Congreso Estatal Isonomía sobre Identidad de Género vs. Identidad Sexual. Castelló de la Plana: Universitat Jaume; 2008. p. 90-3.

Recibido el 12/Sep/2012

Versión final presentada el 17/Dic/2012

Aprobado el 08/Ene/2013 